\title{
Clinical Trial Publication Policy
}

National Cancer Institute

\section{Source}

National Cancer Institute. Clinical Trial Publication Policy. NCI Thesaurus. Code C115557.

The approved guidelines governing the publication of results from a clinical trial. 